\title{
Technical and Economic Evaluation of the Electric Vehicle Charging Network Planning Scheme
}

\author{
Junhai Wang, Lingling Ming, and Dayang Yu
}

\begin{abstract}
Electric vehicle (EV) is an important solution for the traffic and environment problems in cities. However, to plan and build the energy supply and service network is always a challenging work all over the world. This paper is based on the planning of a real charge-swap service network for the electric vehicle in Hangzhou city, China. First, this paper defined the service radius for a network and developed the technical evaluation model; second, the economic evaluation model is proposed based mainly on the investment analysis considering the operating cost and earnings of the network. A case study is presented to show the application of the proposed evaluation method for a real charge-swap service network with a three-hierarchy structure.
\end{abstract}

Index Terms-Electric vehicles, planning, technical evaluation, economic evaluation.

\section{INTRODUCTION}

At present, EVs have not been used widely in China and the charge-swap facilities are severely lacking. But China will make breakthrough in the near future. Reference [1] analyzed the application of pure-electric vehicles in techno-economy based on life-cycle cost theory, and compared pure-electric vehicles with traditional oil-fuelled vehicles. In paper [2], the author proposed the economic and environmental impacts of regional widespread use of EV and offered models to analyze how economically and environmentally sound for a region to develop EVs. Reference [3] described the present construction situation of charging facilities as well as its development plan. The paper will develop technical and economic evaluation model to measure the economic benefit that the charge-swap service network provide for the service company and the service performance that the charge-swap service network provide for EVs users.

\section{Charging Network PlanNing Evaluation FACTOR ANALYSIS}

\section{A. Introduction of Charging Infrastructure}

There are four types of facilities in charge-swap service network: charging pile, battery-charging and battery-swapping station(CSS), centralized battery-charging station(CCS) and battery-swapping station(BSS), as

Manuscript received February 2, 2014; revised June 24, 2014.

Junhai Wang is with Hangzhou Power Supply Company in Zhejiang Provincial Electric Power Company, Hangzhou, China (e-mail: wanghdtv@263.net).

Lingling Ming and Dayang Yu are with Laboratory for the Synergistic Dispatch of the Decentralized Electric Power Grid, Shandong University, Jinan, China (e-mail: mingling0104@163.com, yudayang@sdu.edu.cn). described next.

Electric vehicles which load chargers use the AC power for electricity by charging piles and the rated power is under $5 \mathrm{KW}$. Generally speaking, charging piles are mainly installed on parking spaces such as underground garage of residential areas, public parking lot and shopping mall.

CSS swap fully charged batteries for depleted ones for electric vehicles and eliminate much of the waiting time. The time of the process is only few minutes. CSS can charge the depleted batteries collectively when the grid load is low and can preserve batteries. There are three types of CSS based on the scale of the size of CSS: 100 chargers stations, 200 chargers stations and 400 chargers stations. If it's 100 chargers station, the station is equipped with 100 chargers to charge batteries; 200 chargers station, the station is equipped with 200 chargers; 400 chargers station, the station is equipped with 400 chargers, and so on. The chargers can charge batteries 5 times every day.

CCS and BSS provide energy services for electric vehicles together. CCS charges depleted batteries centrally and carries fully charged batteries for BSS 4 times every day. Then BSS swap fully charged batteries for depleted ones for electric vehicles. According to the experience of electric vehicle market, CCS is usually equipped with 3000 chargers and usually charge batteries 2 times every day. A CCS usually carry batteries for 20 25 BSSs. CCS can carry 80 batteries for BSS one time and BSS is supplemented with 4 carrier vehicles to carry batteries.

\section{B. Technology and Structure of Charging Network}

Charging facility network has the mode of battery swapping as the threads of technology. The paper presents the EV charging facility network planning aimed at vehicles which get energy by battery replacement.

There are tow types of charge-swap service network in the paper: the two-tier structure service network and the three-tier structure service network. The two-tier structure service network is composed of charging pile and CSS and the three-tier structure service network is composed of charging pile, CSS, CCS and BSS. The three-tier structure service network is developed from the two-tier structure service network.

\section{Technical Evaluation Model of Charge-SwaP SERVICE NETWORK}

The paper has the full-network service capability and the service radius of the service network as the technical evaluation indicators. The service ability of the whole network should not be less than the charge-swap demand of 
EVs. The rationality of charge-swap service network is related to the service radius. The service radius of charge-swap service network must be less than the driving range of Evs [4]. The service radius should ensure that the service radius is less than $10 \%-20 \%$ of the driving range of EVs [5].

\section{A. The Service Ability of Charge-Swap Service Network}

The service ability of charge-swap service network depends on the type and the number of charge-swap facility and the full-network charge-swap service utilization coefficient. The charge-swap facility service network faces the problem of shortage of service supply because of the unreasonable layout of charging facilities from the whole service network point. So the paper cites the full-network service utilization coefficient to reduce the full-network service capability.

$$
N_{H}=\sum_{\mathrm{i}=0}^{n} F_{H} \times \lambda
$$

where $N_{H}$ represents the actual full-network service ability of battery replacement; $F_{H}$ represents actual service ability of single station; $\lambda$ denotes the full-network service utilization coefficient of the battery swapping service and $n$ denotes the number of charge-swap facilities.

\section{B. The Service Radius of Charge-Swap Service Network}

The service radius of charge-swap service network should meet the code for transport planning on urban road [6]. The service radius is described below.

$$
R=\sqrt{S / n / 3.14}
$$

where $R$ represents the service radius of charge-swap service network; $S$ denotes the area of planned district and $n$ denotes the number of charge-swap facilities.

\section{ECONOMIC EVALUATION MODEl OF CHARGE-SWAP SERVICE NETWORK}

The paper analyzes the life-cycle cost of charge-swap service network. The life-cycle cost of charge-swap service network mainly concludes battery investment, infrastructure investment, associated power network investment, equipment maintenance cost, battery transportation cost and labor cost [7].

\section{A. Charge-Swap Service Network Investment Analysis}

The battery investment is described below.

$$
S_{d}=\sum_{i=1}^{n_{c l x}} 4 \times C_{S} \times r \times P .
$$

where $S_{d}$ represents battery investment; 4 denotes 4 denotes 4 batteries loaded in a electric vehicle; $C_{S}$ denotes the number of one type of electric vehicle; $r$ represents the redundancy of the battery; $P$ denotes the cost of every battery and $n_{c l x}$ denotes the number of the kinds of electric vehicles.
The equipment maintenance cost of every facility accounts for 2 percent of the investments of the infrastructure and the associated power network. The battery transportation cost every time is 400 yuan, and the total transportation cost every year is 584000 yuan every BSS because that the CCS need to transport batteries to the BSS 4 times every day.

The staff assignment of every facility is shown in Table I.

TABLE I: THE STAFF ASSIGNMENT OF EVERY FACILITY

(a)

Facility

(a) The number of workers 12

(b) \begin{tabular}{cccc}
\hline \hline 200chargers station & 400chargers station & CCS & BSS \\
\hline \hline
\end{tabular}

Every employee is engaged at a salary of 50000 yuan a year.

According to the present empirical data, the investments of every facility are presented. (Shown in Table II).

TABLE II: THE INVESTMENTS OF EVERY FACILITY

(a)

\begin{tabular}{ccc}
\hline Facility & \multicolumn{2}{c}{ CSS } \\
\cline { 2 - 4 } & \multicolumn{2}{c}{ 100 chargers station } \\
\hline Infrastructure investment(million) \\
Associated power network investment \\
(million) \\
Equipment maintenance cost(million) \\
Labor cost(million)
\end{tabular}

\section{B. Charge-Swap Service Earnings Analysis}

The EV service companies charge the user of EV by the mileage because the mileage of electric vehicle is predictable.

$$
Z=\sum_{i=1}^{n_{c k}} C_{S} \times M \times R \times \partial
$$

where $Z$ represents the earning every year; $C_{S}$ denotes the number of one type of electric vehicle; $M$ represents the mileage of electric vehicle every day; $R$ denotes the number of working days every year; $\partial$ denotes the service price per kilometer and $n_{c l x}$ denotes the number of type of electric vehicles.

\section{Operation Benefits Analysis of Charge-Swap Service Network}

The economic evaluation indicator can analyze and evaluate the charge-swap service network planning. The paper has the internal rate of return (IRR) and dynamic investment pay-back period as the economic evaluation indicators.

The net present value(NPV) is the present value of current and future benefit minus the present value of current and future costs. 


$$
N P V=\sum_{t=0}^{N} \frac{C F_{t}}{(1+r)^{t}}
$$

where $C F_{t}$ represents the expected net cash flow; $N$ denotes the lifecycle of the investment project and $r$ represents the discount rate.

If the NPV is positive, the project can add shareholder value, or the project can reduce shareholder value.

Internal rate of return (IRR) is the discount rate that equates the present value of overall capital inflows to the present value of overall capital outflows. If the IRR of a project is greater than the rate of return required by EV service company, service providers should invest in the project, or service providers should not invest in the project [8].

$$
0=C F_{0}+\frac{C F_{1}}{1+I R R}+\frac{C F_{2}}{(1+I R R)^{2}}+\ldots+\frac{C F_{N}}{(1+I R R)^{N}}
$$

Dynamic investment pay-back period is the time that the net income of the project will take to compensate for the entire investment. It is an indicator that can assess the recycling capacity of project investment.

$$
P_{t}=N_{z}-1+\frac{Z_{l}}{Z_{j}}
$$

where $P_{t}$ represents the dynamic investment pay-back period; $N_{z}$ denotes the year when the positive present value of cumulative net cash flow appears; $Z_{l}$ represents the present value of net cash flow in the year before $N_{z}$ and $Z_{j}$ denotes the present value of net cash flow in $N_{z}$ year.

\section{CASE STUdy}

The paper studies the technical and economic evaluation of the EV charging network planning scheme in Hangzhou city according to the situation of EV and charge-swap facility. The paper makes a three-tier structure service network planning scheme as an example. There are 3 types of EVs in the paper: taxi, official vehicle and private car. The situation of EV and charge-swap facility is described below.

TABLE III: THE PRESENT RULE OF EV OPERATION

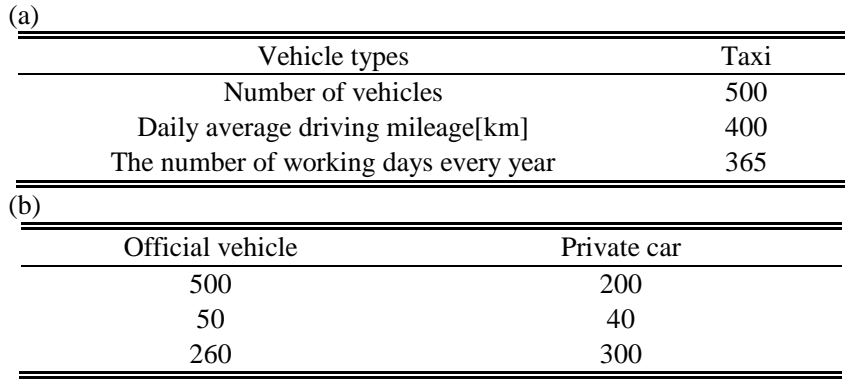

The number of every kind charge-swap facility is decided according toTable III.

\section{A. Technical Evaluation of the Service Network}

The three-tier structure service network planning scheme can supply service for 3000 EVs every day. The area of planned district is 700 square kilometers and the service radius is 2.5 kilometers.

\section{B. Economic Evaluation of the Service Network}

The paper uses redundancy to increase the number of batteries in order to ensure that battery can meet the charging demand of EVs. The redundancy of taxi, official vehicle and private car are 2, 1.2 and 1.2, respectively. According to the current data, the price of a battery is 7500 yuan considering the government subsidy. The battery investment is 55.2 million in the first year. At present, the life of the battery is 5 years, so batteries should be exchanged in the sixth year. The paper supposes that the price of a battery will increase to 15000 yuan when the government subsidy is canceled according to the developing trends of battery industry. The battery range will increase to double its original one and the charge-swap demand will be reduced by half. The battery investment is 110.4 million in the sixth year. Every charge-swap facility will reduce 3 workers in the sixth year because that the workers can handle the machine skillfully. The every investment in construction period is decided according toTable I,Table II and Table IV.

TABLE IV: The ThreE-TIER Structure SERVICE Network PlanNing SCHEME

(a)

\begin{tabular}{lccc}
\hline \hline \multicolumn{1}{c}{ Facility } & 100chargers station \\
\hline \multicolumn{3}{l}{ The number of Facility } & 4 \\
\hline \hline (b) & & & \\
\hline \hline 200chargers station & 400chargers station & CCS & BSS \\
\hline 6 & 2 & 1 & 22 \\
\hline \hline
\end{tabular}

TABLE V: ESTIMATES OF EVERY INVESTMENT IN CONSTRUCTION PERIOD (a)

\begin{tabular}{cccc}
\hline \hline Investment types & Battery investment & $\begin{array}{c}\text { Infrastructure } \\
\text { investment }\end{array}$ \\
\hline \hline Investments & \multicolumn{2}{c}{55.2} & 238 \\
\hline (b) & & & \\
\hline \hline $\begin{array}{c}\text { Associated power } \\
\text { network investment }\end{array}$ & $\begin{array}{c}\text { Equipment } \\
\text { maintenance } \\
\text { cost }\end{array}$ & $\begin{array}{c}\text { Battery } \\
\text { transportation } \\
\text { cost }\end{array}$ & $\begin{array}{c}\text { Labor } \\
\text { cost }\end{array}$ \\
\hline 21.6 & 2.61 & 12.85 & 15.9 \\
\hline \hline
\end{tabular}

The service price per kilometer is 0.5 yuan in the first five years of the lifecycle of the project and the earning is 40.95 million every year. The service price is 0.7 yuan in the rest period and the earning is 114.66 million every year.

TABLE VI: CASH Flow EVERY YeAR IN THE LIFECYCLE OF THE PROJECT [MILLION]

\begin{tabular}{ccccc}
\hline \hline Years & First & Second & Third & Fourth \\
\hline Total investment & 314.8 & 0 & 0 & 0 \\
Earnings & 40.95 & 40.95 & 40.95 & 40.95 \\
Costs & 31.35 & 31.35 & 31.35 & 31.35 \\
Taxes & 2.457 & 2.457 & 2.457 & 2.457 \\
Net cash flow & -307.66 & 7.14 & 7.14 & 7.14 \\
Present value of & -307.66 & 6.74 & 6.35 & 5.99 \\
net cash flow & & & & \\
Present value of & & & -294.57 & -288.58 \\
cumulative net & -307.66 & -300.92 & & \\
cash flow & & & & \\
\hline \hline
\end{tabular}


(b)

\begin{tabular}{cccccc}
\hline \hline Fifth & Sixth & Seventh & Eighth & Ninth & Tenth \\
\hline 0 & 110.40 & 0 & 0 & 0 & 0 \\
40.95 & 114.66 & 114.66 & 114.66 & 114.66 & 114.66 \\
31.35 & 26.10 & 26.10 & 26.10 & 26.10 & 26.10 \\
2.457 & 6.8796 & 6.8796 & 6.8796 & 6.8796 & 6.8796 \\
7.14 & -28.72 & 86.18 & 81.68 & 81.68 & 81.68 \\
5.66 & -21.64 & 57.57 & 54.32 & 51.24 & 48.34 \\
-282.92 & -304.38 & -246.81 & -192.48 & -141.24 & -92.89 \\
\hline \hline
\end{tabular}

\begin{tabular}{ccccc} 
(c) & & & & \\
\hline \hline 0 & Twelfth & Thirteenth & Fourteenth & Fifteenth \\
\hline 114.66 & 0 & 0 & 0 & 0 \\
26.10 & 26.14 .66 & 114.66 & 114.66 & 114.66 \\
6.8796 & 6.8796 & 6.8796 & 6.8796 & 6.8796 \\
81.68 & 81.68 & 81.68 & 81.68 & 81.68 \\
45.61 & 43.03 & 40.59 & 38.29 & 36.13 \\
-47.29 & -4.26 & 36.34 & 74.63 & 110.75 \\
\hline \hline
\end{tabular}

The IRR of the planning scheme is $9.57 \%$ and the dynamic investment pay-back period is 11.89 years. (Shown in Table V and Table VI).

\section{CONCLUSION}

The service radius is 2.5 kilometers and The IRR of the planning scheme is $9.57 \%$. The planning scheme is executable. The EV service company can adjust the constrution of the charge-swap service network according to the technical and economic evaluation model. The construction of charge-swap service network should combine the profitability with the the actual operating situation in the future. There are several further work directions from this research. Firstly, the evaluation model would be more accurate if more EV charge-swap facilities are taken into account. Secondly, the evaluation model would think about the environmental factors.

\section{ACKNOWLEDGMENT}

The author thanks the Hangzhou Power Supply Company in China for the support on this study and for the further cooperation.

\section{REFERENCES}

[1] Z. Sui and Z. Wang, "Technical and economic analysis of pure-electric vehicles based on the life-cycle cost theory," in Proc. 2011 IEEE International Conference on Business Management and Electronic Information (BMEI), 2011, vol. 1, pp. 125-129.

[2] L. Deng, Y. Zhang, and W. Liu, "Economic and environmental impacts analyses of regional widespread use of electric vehicles," Research Journal of Applied Sciences, Engineering and Technology, vol. 6, no. 15, pp. 2693-2698, 2013.

[3] X. P. Ju, K. Y. Jiang, and B. Wang, "Electric vehicles and charging networks in China," in Proc. 2011 4th IEEE International Conference on Power Electronics Systems and Applications (PESA), 2011, pp. 1-6.

[4] S. Shahidinejad, S. Filizadeh, and E. Bibeau, "Profile of charging load on the grid due to plug-in vehicles," IEEE Transactions on Smart Grid, vol. 3, issue 1, pp. 135-141, 2012.

[5] L. M. Zhou, "Study of electric vehicles charging station location planning," dissertation, Shandong University, 2012, pp. 46-47.

[6] M. Cetin and G. F. List, "Integrated modeling of information and physical flows in transportation systems," Transportation Research Part C: Emerging Technologies, vol. 14, no. 2, pp. 139-156, 2006.

[7] B. A. Hamilton, American System Engineering Management, Beijing: Aviation Industry Press, 1991.

[8] C. B. Xu and Q. Chen, "Comparative study on IRRs for financial analysis of investment projects," Energy Technology and Economics, vol. 23 , no. 10 , pp. 1-6, 2011.

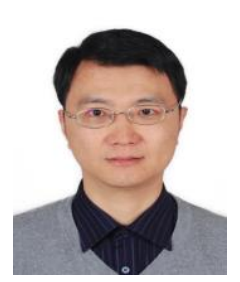

Junhai Wang was born in Zhejiang Province, China, in 1971. He obtained his B.Sc. and M.Sc. degrees from Zhejiang University in motor and control.

$\mathrm{He}$ is a senior engineer in Hangzhou Power Supply Company in Zhejiang provincial electric power company, Hangzhou, China. His research interests include power grid planning and design management and electric vehicle charge-swap facilities operation management.

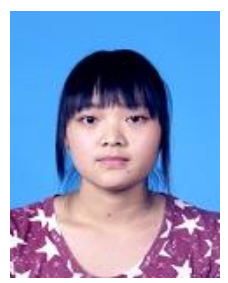

Lingling Ming was born in Taian Province, China, in 1989. She obtained her B.Sc. degree from University of Jinan in 2012. She is currently pursuing the M.Sc. degree with School of Electrical Engineering, Shandong University, Jinan, China.

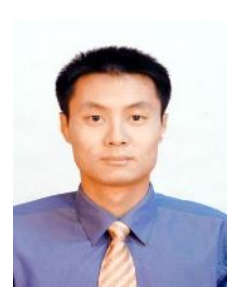

Dayang $\mathbf{Y u}$ is an associate professor worked in Laboratory for the Synergistic Dispatch of the Decentralized Electric Power Grid, School of Electrical Engineering, Shandong University, Jinan, China. 